\title{
Actitudes de los alumnos de Primaria y Secundaria ante la visión dicotómica de la Ciencia
}

\section{Primary and Secondary students' attitude towards the dichotomic view of Science}

\author{
A. de Pro Bueno, A. Pérez Manzano \\ Universidad de Murcia \\ nono@um.es,aperez@um.es
}

RESUMEN • La formación de las actitudes de los alumnos hacia la ciencia y la tecnología (CyT) está influida por muchos factores: la forma de trabajar estas en la escuela, la percepción social de las aportaciones científicas y tecnológicas, el enfoque de las noticias sobre los descubrimientos en los medios de comunicación, el uso de ambas por la publicidad... Sin embargo, si analizamos detenidamente la imagen que se trasmite de la CyT, podemos apreciar ciertos matices dicotómicos: en ocasiones, se muestra la "ciencia buena», avances médicos, aparatos para mejorar la calidad de vida, alargamiento de la esperanza de vida, etc.; pero en otras aparece la "ciencia mala», la asociada a la contaminación o a las radiaciones, la que hay detrás de la industria militar, la que produce efectos nocivos sobre la salud y el medio ambiente... En el presente artículo se pretende explorar el posicionamiento de los alumnos de primaria y secundaria ante esta visión dicotómica de la ciencia.

PALABRAS CLAVE: actitudes hacia la Ciencia; Ciencia y sociedad; currículo; educación Primaria; educación Secundaria.

ABSTRACT • The formation of student attitudes towards science and technology (S\&T) is influenced by many factors: The way they are worked in school, the social perception of scientific and technological contributions, the focus of the news about discoveries in the media, the use of both by advertising... However, if we look thoroughly at the image that is transmitted from the S\&T, we see certain dichotomous issues: sometimes it is shown the "good science», medical advances, devices which are able to improve life quality, lengthening life expectancy, and so on; but in others it appears the "bad science», the one linked to pollution or radiation or behind the military industry, or the one which produces harmful effects to health and environment, etc. The intention of this article is to explore the attitude of elementary and secondary education students against this dichotomic view of science.

KEYWORDS: attitudes towards Science; Science and society; curriculum; elementary education; secondary education.

Fecha de recepción: septiembre 2012 • Aceptado: abril 2013

de Pro Bueno, A., Pérez Manzano, A. (2014) Actitudes de los alumnos de Primaria y

Secundaria ante la visión dicotómica de la Ciencia. Enseñanza de las Ciencias, 32.3,

pp. 111-132 


\section{INTRODUCCIÓN}

Ya en 1935 Gordon Allport decía que resultaba más sencillo medir las actitudes que definirlas (Allport, 1935). Más recientemente, Osborne, Simon y Collins (2003) o Kind, Jones y Barmby (2007) seguían señalando esta dificultad. Pero, con o sin una definición compartida, podemos aceptar que las actitudes hacia la ciencia no solo pueden condicionar el rendimiento escolar en unas asignaturas determinadas, sino la forma de pensar y hacer de la sociedad (la ciencia es una forma de cultura), su imagen social (y, en consecuencia, al apoyo socioeconómico a las investigaciones y programas científicos) o la cantidad de investigadores o profesionales en este campo del conocimiento.

Según puso de manifiesto el Informe de la Comisión Europea (European Comision, 2004), las consecuencias sociales y educativas de la adquisición de actitudes hacia las ciencias y hacia las materias de carácter científico resultan importantes... y preocupantes. En este sentido y en relación con nuestro contexto educativo, compartimos muchas de las reflexiones realizadas al respecto por Vázquez, Acevedo y Manasero (2005). Creemos que son necesarias investigaciones que nos permitan un diagnóstico profundo de la situación, como paso previo a la realización de actuaciones intencionadas para mejorarla.

No obstante, la investigación en este ámbito no es nueva. De hecho, la gran producción científica generada ha sido analizada por algunos autores (por ejemplo, Vázquez y Manasero 2007a y 2007b) y nos ha llevado a algunos hallazgos:

a) Las actitudes hacia la ciencia suelen empeorar con el nivel educativo (Pell y Jarvis, 2001; Gibson y Chase, 2002; Murphy y Beggs, 2003; Lindahl, 2005; George, 2006; Barmby, Kind y Jones, 2008; Vázquez y Manassero, 2008; Marbá y Márquez, 2010 ...) y ello afecta a todas las asignaturas de carácter científico, pero especialmente a la física y química (según la revisión realizada por Vázquez y Manasero [2007b] o el trabajo de Marbá y Márquez [2010]).

b) Se ha estudiado su dependencia del género, probablemente para tratar de explicar el volumen desigual de vocaciones en las carreras científico-tecnológicas; parece que a veces se han impuesto estereotipos, tópicos y tradiciones sociales a la hora de vincular determinadas profesiones a esta variable (Murphy y Beggs, 2003; Osborne, Simon y Collins, 2003; Sjøberg y Schreiner, 2005; Jenkins y Pell, 2006; Vázquez y Manassero, 2007a, 2008, 2009; Marbá y Márquez, 2010, etc.).

c) El proyecto ROSE (Schreiner y Sjøberg, 2004), además de reforzar algunos de los hallazgos anteriores, muestra "otras tendencias», como por ejemplo que existe una relación inversa entre el grado de desarrollo del país y las actitudes positivas hacia la ciencia en jóvenes estudiantes de secundaria (Sjøberg, 2000; Sjøberg y Schreiner, 2005 y 2007). Por otro lado, el trabajo de Gil (2012) pone de manifiesto que existen diferencias entre las comunidades autónomas en relación con las actitudes. Es decir, parece que puede haber otros factores -además del nivel educativo y el género- que inciden en la visión del alumnado o en su interés por las ciencias.

d) Existen niveles bajos de confianza en la ciencia respecto a temáticas como la salud, el medio ambiente, etc. (Acevedo, 2005; Vázquez y Manasero, 2008 y 2009; Gil, 2012), lo que nos lleva a situaciones preocupantes cuando se deben enfrentar las consecuencias positivas y negativas de la ciencia y la tecnología, desde una perspectiva social, como es el caso que nos ocupa.

e) Por último, nosotros también hemos realizado aportaciones en el ámbito de las actitudes. Así, por ejemplo, entre las últimas publicadas podríamos citar el estudio sobre la percepción de los niños y adolescentes hacia los científicos y su trabajo (Pro, Pérez y Tárraga, 2009); el trabajo sobre lo que piensan los escolares en la educación obligatoria sobre las asignaturas de ciencias (Pro y Pérez, 2013), e incluso hemos encontrado que los efectos de las propuestas innovadoras desde la perspectiva actitudinal no son conjuntos: se pueden producir cambios significativos en unas actitudes y en otras no (López, Pro y Saura, 2012). 
Sin embargo, en los estudios mencionados, muchas veces se entremezclan resultados obtenidos respecto a la visión de la ciencia y los trabajos de los científicos y los referidos a las distintas materias escolares. Al respecto, hemos señalado y justificado en otro trabajo (Pro, 2003) que existen diferentes variantes en relación con las actitudes: hacia la ciencia y sus descubrimientos, hacia los científicos y su forma de trabajo (en una actividad científica), hacia las asignaturas de ciencia, hacia la creación de hábitos saludables, hacia la conservación del medio ambiente... Para un conocimiento más profundo de estas, parece necesario separar los hallazgos, aunque puedan existir relaciones -e incluso causalidades- entre sus resultados.

Por otro lado, el hecho de que el proyecto ROSE tenga un enfoque transnacional supone implícitamente que existe una cierta dependencia contextual de las actitudes con los sistemas educativos o con las realidades sociales; de hecho, Sjøberg y Schreiner (2010) lo han puesto de manifiesto al estudiar comparativamente los resultados obtenidos en 34 países. Los que este estudio atribuye a España, se han obtenido a partir de la aplicación de los instrumentos ROSE a una amplia muestra de cuarto de la ESO de las Islas Baleares (Vázquez y Manasero, 2007a y 2009). Para nosotros, se aporta un diagnóstico muy interesante; de hecho, como se verá, es el referente prioritario a la hora de discutir nuestros resultados. Ahora bien nuestro estudio ha ampliado la población objeto de estudio a todo el Estado español.

Además, algunos trabajos ya mencionados - por ejemplo, los de Pell y Jarvis (2001), Murphy y Beggs (2003) o Barmby, Kind y Jones (2008) - apuntan a que, al finalizar la educación primaria, parece producirse un punto de inflexión en cuanto a las actitudes. Este nivel educativo ha tenido menos atención en los estudios nacionales e internacionales consultados y creímos que podría ser de gran utilidad disponer de una información representativa de nuestro contexto.

Por último, creemos que puede existir una cierta dependencia de los resultados con la forma de preguntar. Por ello, en este trabajo, que forma parte de una investigación más amplia (Pérez, 2013), nos hemos ocupado de la actitud de los estudiantes cuando se les plantean posiciones dicotómicas hacia las ciencias y el trabajo de los científicos.

\section{LAS ACTITUDES HACIA LAS CIENCIAS EN LOS PROGRAMAS OFICIALES}

Al ampliar la educación obligatoria hasta los 16 años, se realizó una reforma profunda de nuestro sistema educativo. Los cambios afectaron a su estructura, a las competencias del profesorado, a su formación... y, por supuesto, a los programas oficiales (MEC, 1992a y 1992b). Se incorporaron logros de la investigación e innovación de aquel momento; se planteó un currículo semiabierto que debía concretarse en los centros y en las aulas; se defendía el principio de diversificación y la atención a la diversidad; se incluían áreas trasversales que debían impregnar todas las materias; se apostaba por una enseñanza con un enfoque constructivista; se apuntaba a una evaluación formativa y no centrada solo en el rendimiento del alumnado; se distinguían tres tipos de contenidos: conceptos, procedimientos y actitudes; etc.

En relación con este último elemento innovador, aunque las actitudes habían estado presentes en programas oficiales anteriores, una referencia tan directa a su aprendizaje y el hecho de situarlos al mismo nivel que los otros supusieron un salto importante - por lo menos «sobre el papel»- en su reconocimiento en la enseñanza de las ciencias. Además, a pesar de las continuas reformas y contrarreformas, tan habituales en nuestro sistema educativo, su presencia se ha mantenido, unas veces como tipo de contenido y otras entre las competencias que debe adquirir el alumnado.

Así, por ejemplo, si nos centramos en el currículo LOGSE que habían tenido los participantes en nuestra experiencia en el momento en que se realizó el trabajo, en los cuadros 1 y 2 se recogen las actitudes hacia la ciencia (dejamos a un lado los demás ámbitos de este constructo); los dígitos corresponden al bloque y apartado educación primaria y ESO. Hemos diferenciado entre los contenidos favorables (valoración positiva de la ciencia) y desfavorables (actitud crítica o de cautela hacia la ciencia). 
Cuadro 1.

Contenidos de actitudes positivas hacia las ciencias en la LOGSE

\begin{tabular}{|l|}
\hline Valoración positiva de las aportaciones de la ciencia \\
\hline Programa oficial de educación primaria (MEC, 1992a): \\
- Valoración de los distintos trabajos y profesiones y de su función complementaria en el conjunto de la sociedad (6.2) \\
- Valoración de la influencia del desarrollo tecnológico en las condiciones de vida y en el trabajo (6.4) \\
- Valoración de los aparatos y máquinas del entorno habitual como construcciones humanas destinadas a satisfacer las \\
necesidades de las personas y a mejorar su calidad de vida (7.2) \\
- Valoración del impacto del desarrollo tecnológico sobre la evolución de los medios de comunicación y trasporte (9.3) \\
\hline Programa oficial de la ESO (MEC, 1992b): \\
- Reconocimiento de la importancia de los modelos y de su confrontación con los hechos empíricos (1.1) \\
- Valoración de la provisionalidad de las explicaciones como elemento diferenciador del conocimiento científico y como \\
base del carácter no dogmático y cambiante de la ciencia (1.2) \\
- Valoración de la energía en las actividades cotidianas y de su repercusión en la calidad de vida y el desarrollo económico \\
- 2.1 ) \\
- Reconocimiento y valoración de la importancia de los fenómenos ondulatorios en la civilización actual y de la trascen- \\
- Vencia de sus aplicaciones en diversos ámbitos de la actividad humana (2.2) \\
de materiales con nuevas propiedades y el incremento cualitativo y cuantitativo en la producción de alimentos y me- \\
dicinas (3.1) \\
- Valoración de la actitud de perseverancia y riesgo del trabajo de los científicos para explicar los interrogantes que se \\
plantea la humanidad (4.1) \\
- Reconocimiento y valoración de la importancia de la electricidad para la calidad de vida y el desarrollo industrial y \\
tecnológico (11.3)
\end{tabular}

\section{Cuadro 2.}

Contenidos de actitudes de cautela hacia las ciencias en la LOGSE

\begin{tabular}{|c|}
\hline Cautela ante las aportaciones de las ciencias \\
\hline Programa oficial de educación primaria (MEC, 1992a): \\
- Actitud crítica ante las informaciones recibidas a través de los distintos medios de comunicación (9.1). \\
- [...] Actitud crítica ante sus utilizaciones [del ordenador] en la vida cotidiana (9.5) \\
\hline Programa oficial de la ESO (MEC, 1992b): \\
- Valoración crítica del efecto de los productos químicos presentes en el entorno sobre la salud, la calidad de vida, el pa- \\
trimonio artístico y el futuro de nuestra civilización, analizando a su vez las medidas internacionales que se establecen \\
a este respecto (3.1) \\
- [...] rechazo de las actividades humanas contaminantes [del aire] (5.1) \\
- [...] necesidad de recuperar las zonas deterioradas por una previa explotación industrial (5.3) \\
- Defensa del medio ambiente [...], ante actividades humanas responsables de su contaminación y degradación (9.2) \\
\hline
\end{tabular}

No queremos entrar en la puesta en práctica de los programas oficiales; ya lo hemos hecho en otros trabajos, en los que valoramos -entre otros aspectos- la presencia de las actitudes en los proyectos curriculares de los centros o en los libros de texto (Pro, 2006 y 2007; Pro, Sánchez y Valcárcel, 2009). Pero lo cierto es que legalmente estos contenidos han formado parte de los conocimientos científicos que deberían haberse enseñado durante la educación obligatoria, probablemente porque se consideran que forman parte de las necesidades formativas de los ciudadanos. No solo deberían ser una consecuencia descontrolada de la educación recibida fuera de las aulas.

Pero, además, se puede constatar que el propio currículo oficial favorece una visión dicotómica de las ciencias. Hay contenidos en los que se resaltan sus repercusiones positivas (satisfacer necesidades de la humanidad, mejorar la calidad de vida, producir alimentos y medicinas, etc.) y en otros se «habla 
con cautela» de sus consecuencias (efectos negativos en la salud, contaminación, impactos medioambientales, etc.). Se trabajen o no en las clases, el alumnado se encuentra ante unas dicotomías en su vida cotidiana. ¿Qué predomina cuando se les pregunta por ellas? ¿¿Sobreponen las contribuciones a favor a las que se suponen que no son deseables? ¿ $\mathrm{O}$, por el contrario, las suspicacias o cautelas son más sólidas que sus efectos favorables?

Hemos tratado de encontrar respuestas a estas cuestiones. Por ello, nos planteamos los siguientes problemas principales (PP):

PP1. ¿Qué posiciones tienen los niños y adolescentes españoles ante una serie de dicotomías relacionadas con los productos de la ciencia y sus repercusiones sociales?

PP2. ¿Dependen los posicionamientos del nivel educativo, el género o el tipo de centro?

\section{METODOLOGÍA DE LA INVESTIGACIÓN}

\section{Selección y descripción de la muestra}

En un trabajo de carácter demoscópico, como éste, necesitamos definir la población. Ésta se basó en los datos oficiales del Ministerio de Educación, Cultura y Deporte correspondientes al curso 2002-2003. Se eligieron los últimos cursos de cada etapa -sexto de EP y cuarto de la ESO- ya que eran los niveles más adecuados para nuestros propósitos.

Los dos niveles educativos -EP y ESO- se estratificaron por comunidades autónomas y tipo de centro (público y privado). El cruce de las tres variables generó 68 estratos que se distribuyeron mediante fijación proporcional, según su peso respecto a la población. De esta manera, quedaron determinados los porcentajes de dichos estratos en el total de la muestra.

Luego usamos un muestreo por conglomerados bietápico. Las unidades primarias fueron los centros (controlamos su tamaño, y su localización provincial y comarcal) y las secundarias fueron las aulas (seleccionadas con el criterio de disponibilidad). En los cálculos, se estimó que la asistencia media de alumnos por aula era 18 en educación primaria y 21 en educación secundaria.

Los resultados del peso de los estratos, los números de centros y la distribución de alumnos se han recogido en el anexo 1. En la tabla 1 se recogen algunas características de la muestra.

Tabla 1.

Algunos datos que describen la muestra

\begin{tabular}{|l|c|c|c|c|}
\hline \multirow{2}{*}{} & \multicolumn{2}{|c|}{ Educación primaria } & \multicolumn{2}{c|}{ Educación secundaria } \\
\cline { 2 - 5 } & Número de aulas & Número de alumnos & Número de aulas & Número de alumnos \\
\hline Centros públicos & 165 & 2.591 & 124 & 1.922 \\
\hline Centros privados & 80 & 1.304 & 59 & 1.010 \\
\hline Total de la muestra & 245 & 3.895 & 183 & 2.932 \\
\hline
\end{tabular}

Finalmente, se ha trabajado con 6.827 cuestionarios (3.475 chicos, 3.308 chicas y 44 que no indicaron el género). El error muestral por nivel educativo es del 0,8\% en EP y del 0,9\% en ESO. Para el conjunto de la muestra el error muestral máximo es del 0,01\%.

Como hemos mencionado, con el PP2 pretendemos estudiar la dependencia del nivel educativo, el género y el tipo de centro. Aunque se tuvo en cuenta las comunidades autónomas en la elección de la muestra, no describiremos los resultados en esta variable porque sería discutible el contraste de diferencias dada la heterogeneidad del tamaño de los grupos. También hemos recogido información 
de variables como las profesiones de los progenitores, tamaño del centro o tipo de población pero el estudio de la dependencia de estas la dejaremos para otros trabajos.

\section{Instrumento de recogida de información}

Hemos utilizado el cuestionario PANA (Proyecto de Actitudes hacia las ciencias en Niños y Adolescentes) (Pérez y Pro, 2005), diseñado específicamente para esta investigación.

En primer lugar, realizamos un estudio piloto con una primera versión que fue aplicada a una muestra de alumnos de estas edades. Revisamos la redacción de algunas cuestiones, el orden, las opciones de respuestas, la duración de la prueba, etc. Con todo ello, se realizó una segunda versión que resultó ser la definitiva. Ya hemos comentado que solo nos vamos a centrar en las cuestiones que se refieren a las dicotomías pero el cuestionario contenía más información.

La reproducción de las cuestiones concretas utilizadas en este trabajo se recoge en Pro y Pérez (2013a), pero comentaremos algunas características relevantes. Había cinco ítems que planteaban otras tantas dicotomías. En el cuadro 3 se señalan las visiones positivas y negativas que se enfrentaban, y las opciones de respuesta que se ofrecían.

\section{Cuadro 3.}

Características de los ítems del Cuestionario PANA de este trabajo

\begin{tabular}{|c|c|c|c|c|}
\hline Dicotomía & Visión positiva & Visión negativa & \multicolumn{2}{|c|}{$\begin{array}{l}\text { Opciones de respuestas } \\
\text { (Estoy de acuerdo con...) }\end{array}$} \\
\hline Salud & $\begin{array}{l}\text { La ciencia ha hecho } \\
\text { que mejore la salud: } \\
\text { medicamentos, vacunas, } \\
\text { aparatos para curarnos... }\end{array}$ & $\begin{array}{l}\text { Los científicos han } \\
\text { creado armas para } \\
\text { destruirnos y productos } \\
\text { que matan }\end{array}$ & $\begin{array}{l}\text { - Solo con la visión } \\
\text { positiva } \\
\text { - Más con la positiva } \\
\text { que con la negativa } \\
\text { - Con las dos iguales }\end{array}$ & $\begin{array}{l}\text { - Con ninguna } \\
\text { - Más con la negativa } \\
\text { que con la positiva } \\
\text { - Solo con la visión } \\
\text { negativa }\end{array}$ \\
\hline Estilo de vida & $\begin{array}{l}\text { La ciencia ha hecho que } \\
\text { podamos: oír música, } \\
\text { ver la TV, chatear con el } \\
\text { ordenador, jugar con la } \\
\text { consola, ir de viaje... }\end{array}$ & $\begin{array}{l}\text { La ciencia ha hecho que } \\
\text { la vida sea estresada, } \\
\text { que abunde la comodi- } \\
\text { dad y el individualismo, } \\
\text { haya más aislamiento }\end{array}$ & $\begin{array}{r}\text { - Solo con la positiva } \\
\text { - Más con la positiva } \\
\text { que con la negativa } \\
\text { - Con las dos iguales }\end{array}$ & $\begin{array}{l}\text { - Ninguna de las dos } \\
\text { - Más con la negativa } \\
\text { que con la positiva } \\
\text { - Solo con la negativa }\end{array}$ \\
\hline Noticias & $\begin{array}{l}\text { Hay noticias positivas: } \\
\text { gasóleo que rinde más y } \\
\text { contamina menos, uso } \\
\text { de fuentes alternativas, } \\
\text { fecundación in vitro... }\end{array}$ & $\begin{array}{l}\text { Hay noticias negativas: } \\
\text { producción nubes tóxi- } \\
\text { cas, residuos de fábricas, } \\
\text { agujero de la capa de } \\
\text { ozono... }\end{array}$ & $\begin{array}{l}\text { - Hay más noticias po- } \\
\text { sitivas que negativas } \\
\text { - Hay igual número de } \\
\text { noticias positivas y } \\
\text { negativas }\end{array}$ & $\begin{array}{l}\text { - No sé lo que más } \\
\text { abunda, las positivas } \\
\text { o las negativas } \\
\text { - Hay más noticias ne- } \\
\text { gativas que positivas }\end{array}$ \\
\hline $\begin{array}{l}\text { Credibilidad } \\
\text { información }\end{array}$ & $\begin{array}{l}\text { Las antenas cumplen } \\
\text { las normas de seguridad } \\
\text { y no producen efectos } \\
\text { perjudiciales }\end{array}$ & $\begin{array}{l}\text { Las antenas producen } \\
\text { cáncer }\end{array}$ & $\begin{array}{l}\text { - Solo creo la positiva } \\
\text { - Las dos pueden ser } \\
\text { ciertas } \\
\text { - Ninguna es cierta }\end{array}$ & $\begin{array}{l}\text { - No sé quién tiene } \\
\text { razón } \\
\text { - Solo creo la negativa }\end{array}$ \\
\hline $\begin{array}{l}\text { Repercusión } \\
\text { social }\end{array}$ & $\begin{array}{l}\text { La ciencia es sinónimo } \\
\text { de progreso: internet, } \\
\text { video-consolas, AVE...; } \\
\text { así como de nuevas } \\
\text { profesiones y más opor- } \\
\text { tunidades }\end{array}$ & $\begin{array}{l}\text { Las ciencias provocan } \\
\text { diferencias sociales, los } \\
\text { ricos más ricos y los } \\
\text { pobres más pobres, más } \\
\text { paro porque las máqui- } \\
\text { nas sustituyen mano de } \\
\text { obra... }\end{array}$ & 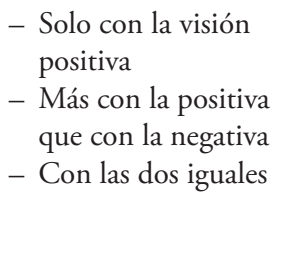 & $\begin{array}{l}\text { - Con ninguna } \\
\text { - Más con la negativa } \\
\text { que con la positiva } \\
\text { - Solo con la visión } \\
\text { negativa }\end{array}$ \\
\hline
\end{tabular}


Para simplificar su denominación a lo largo del trabajo las identificaremos como: «Salud « (mejora de la salud frente a industria militar), «Estilo de vida» (mejora estilo de vida frente a actitudes no deseables), «Noticias» (noticias positivas frente a noticias negativas), «Credibilidad información» (versión favorable frente a versión desfavorable) y «Repercusión social» (avances sociales frente a problemas sociales). Puede comprobarse que estas dicotomías están implícitas en los programas oficiales (cuadros 1 y 2 ).

Había una sexta cuestión en la que debían hacer una valoración global de las contribuciones de las ciencias («Valoración global»); en ella debían optar por: «Solo han hecho aportaciones positivas», «Han hecho más positivas que negativas», «No sé si han hecho más positivas que negativas», "Han hecho más negativas que positivas» $\mathrm{o}$ «Solo han hecho negativas». También debían justificar su elección, pero en general usaron como argumentos el contenido de las dicotomías anteriores, es decir, no realizaron aportaciones propias o diferentes a las ya señaladas. Por ello, estas explicaciones no se describirán ni analizarán en los resultados que recogemos en este trabajo.

Como puede verse, hemos tratado de variar las opciones de respuesta manteniendo un mismo estilo. La razón es que, en este tipo de cuestionarios, el alumnado procede con un "cierto automatismo» si las opciones son siempre las mismas. Cuando se modifican, parece que se favorece que las contestaciones sean más reflexivas.

\section{Aplicación del cuestionario}

El Cuestionario PANA fue pasado entre marzo y junio de 2004. Para llevar a cabo nuestra tarea, contactamos con unos coordinadores de zona de todo el Estado español. Ellos se encargaron de seleccionar los centros (unidades primarias de muestreo) y las aulas (unidades secundarias). Las instrucciones que se les enviaron se recogen en Pro y Pérez (2013b).

La aplicación del cuestionario fue realizada por más de 40 profesores - universitarios, de educación secundaria y maestros- de las diferentes comunidades autónomas. Todos usaron el mismo protocolo (presentación del cuestionario, observaciones y explicaciones). A la vista de la información recogida del proceso seguido, podemos decir que no hubo incidencias que afectaran a las respuestas que hemos analizado.

\section{Tratamiento de la información}

La tabulación de los datos y el análisis de estos se realizaron con el paquete estadístico SPSS 15.0 para Windows. Como veremos en la descripción de los resultados se ha mantenido la literalidad de las opciones que ofrecíamos con el cuestionario PANA. No obstante, para facilitar algunos contrastes, hemos agrupado las opciones favorables (Solo positivas-Más las positivas que...), las neutras, indecisas o no definidas (Igual las positivas y las negativas-Ni las positivas ni las negativas-No lo sé) y las desfavorables (Solo negativas-Más las negativas...).

Las variables de cruce utilizadas en este trabajo han sido: el nivel educativo (sexto de EP y cuarto de la ESO), el género (hombre y mujer) y el tipo de centro (público y privado).

Para tener una visión global de las posiciones creamos una variable -«Total dicotomía»- que representara el conjunto de los posicionamientos de cada alumno a lo largo del cuestionario. Codificamos numéricamente las respuestas de cada estudiante en tres niveles (posición positiva, neutra y negativa) en las cinco dicotomías y mantuvimos los cinco niveles de la variable «Valoración global» («olo positivas», «Más positivas que negativas», "No sé», «Más negativas que positivas», «Solo negativas»). Sumamos los valores obtenidos en los casos en que habían respondido a todos los ítems planteados. De esta manera resultaba un número comprendido entre 0 y 14 . 
Como somos conscientes de los errores que cometeríamos si consideráramos este como un «valor exacto» del posicionamiento, fijamos unos intervalos y etiquetamos los resultados en cinco categorías: "Muy negativa», "Negativa», "Neutra», "Positiva» y "Muy positiva». Estos valores podían representar cuál era la valoración realizada sobre las aportaciones de la ciencia. No obstante, como veremos, el uso de esta variable no ha obviado el análisis de las otras que hemos mencionado.

\section{ANÁLISIS DE LOS RESULTADOS OBTENIDOS}

Teniendo en cuenta los problemas principales de este trabajo de investigación, describimos los resultados globales y posteriormente los obtenidos en los cruces con las variables nivel educativo, género y tipo de centro.

\section{Resultados globales}

Los resultados en cada ítem de las dicotomías planteadas se recogen en la siguiente tabla. Hemos mantenido en la primera columna las opciones de respuesta (con una redacción simplificada). En las siguientes aparecen la frecuencia de cada opción y los porcentajes que suponen en relación con el total de la muestra. El tramado pretende que se perciban mejor las opciones favorables, neutras y desfavorables en cada dicotomía.

Tabla 2.

Resultados en las variables dicotómicas y en la valoración global

\begin{tabular}{|c|c|c|c|c|c|c|}
\hline & $\begin{array}{c}\text { Mejoras } \\
\text { en salud- } \\
\text { Industria } \\
\text { militar }\end{array}$ & $\begin{array}{l}\text { Mejor estilo } \\
\text { de vida- } \\
\text { Actitudes no } \\
\text { deseables }\end{array}$ & $\begin{array}{c}\text { Noticias } \\
\text { positivas- } \\
\text { Noticias } \\
\text { negativas }\end{array}$ & $\begin{array}{l}\text { Credibilidad } \\
\text { (versión } \\
\text { favorable- } \\
\text { versión } \\
\text { desfavorable) }\end{array}$ & $\begin{array}{c}\text { Avances } \\
\text { sociales- } \\
\text { Problemas } \\
\text { sociales }\end{array}$ & $\begin{array}{c}\text { Valoración } \\
\text { global, } \\
\text { aportaciones } \\
\text { de ciencias }\end{array}$ \\
\hline & $\begin{array}{c}\text { Frec. } \\
\%\end{array}$ & $\begin{array}{c}\text { Frec. } \\
\%\end{array}$ & $\begin{array}{c}\text { Frec. } \\
\%\end{array}$ & $\begin{array}{c}\text { Frec. } \\
\%\end{array}$ & $\begin{array}{c}\text { Frec. } \\
\%\end{array}$ & $\begin{array}{c}\text { Frec. } \\
\%\end{array}$ \\
\hline Solo las positivas & $\begin{array}{c}888 \\
13,0 \%\end{array}$ & $\begin{array}{c}764 \\
11,2 \%\end{array}$ & & $\begin{array}{c}528 \\
7,7 \%\end{array}$ & $\begin{array}{c}381 \\
5,6 \%\end{array}$ & $\begin{array}{c}623 \\
9,1 \%\end{array}$ \\
\hline Más las positivas & $\begin{array}{c}2457 \\
36,0 \%\end{array}$ & $\begin{array}{c}1.994 \\
29,2 \%\end{array}$ & $\begin{array}{c}1.599 \\
23,4 \%\end{array}$ & & $\begin{array}{c}1.682 \\
24,6 \%\end{array}$ & $\begin{array}{l}3.526 \\
51,6 \%\end{array}$ \\
\hline $\begin{array}{l}\text { Igual positivas que } \\
\text { negativas }\end{array}$ & $\begin{array}{c}3075 \\
45,0 \%\end{array}$ & $\begin{array}{l}2.490 \\
36,5 \%\end{array}$ & $\begin{array}{c}2110 \\
30,9 \%\end{array}$ & $\begin{array}{c}3724 \\
54,5 \%\end{array}$ & $\begin{array}{l}2.910 \\
42,6 \%\end{array}$ & \\
\hline $\begin{array}{l}\text { Ni positivas ni } \\
\text { negativas }\end{array}$ & $\begin{array}{c}108 \\
1,6 \%\end{array}$ & $\begin{array}{c}144 \\
2,1 \%\end{array}$ & & $\begin{array}{c}646 \\
9,5 \%\end{array}$ & $\begin{array}{c}359 \\
5,3 \%\end{array}$ & \\
\hline No lo sé & & & $\begin{array}{r}1.389 \\
20,3 \%\end{array}$ & $\begin{array}{c}1179 \\
17,3 \% \\
\end{array}$ & & $\begin{array}{c}2.094 \\
30,7 \%\end{array}$ \\
\hline Más las negativas & $\begin{array}{c}171 \\
2,5 \%\end{array}$ & $\begin{array}{l}1.159 \\
17,0 \%\end{array}$ & $\begin{array}{c}1.628 \\
23,8 \%\end{array}$ & & $\begin{array}{c}1.131 \\
16,6 \%\end{array}$ & $\begin{array}{c}350 \\
5,1 \%\end{array}$ \\
\hline Solo las negativas & $\begin{array}{c}53 \\
0,8 \%\end{array}$ & $\begin{array}{c}201 \\
2,9 \%\end{array}$ & & $\begin{array}{c}640 \\
9,4 \%\end{array}$ & $\begin{array}{c}246 \\
3,6 \%\end{array}$ & $\begin{array}{c}89 \\
1,3 \%\end{array}$ \\
\hline No contesta & $\begin{array}{c}75 \\
1,1 \%\end{array}$ & $\begin{array}{c}75 \\
1,1 \%\end{array}$ & $\begin{array}{c}101 \\
1,5 \%\end{array}$ & $\begin{array}{c}110 \\
1,6 \%\end{array}$ & $\begin{array}{c}118 \\
1,7 \%\end{array}$ & $\begin{array}{c}145 \\
2,1 \%\end{array}$ \\
\hline
\end{tabular}


A la vista de los valores obtenidos podemos decir:

- Existen dicotomías - «Salud» y «Estilo de vida»- en las que predominan las opciones positivas -frente a las neutras y negativas- hacia las contribuciones de la ciencia y la tecnología en dichos ámbitos.

- En las otras tres predominan las respuestas equidistantes, con porcentajes sobre el $50 \%$ en «Noticias» y «Repercusiones sociales» y de más del ¡80\%! en «Credibilidad».

- En ninguna de las dicotomías planteadas las valoraciones negativas llegan al 25\%; en dos, no alcanzan el $10 \%$.

- Los porcentajes y las tendencias de las «dicotomías aisladas» no se mantienen en la variable "Valoración global»; en esta, existe un claro predominio de las opciones positivas, aunque una tercera parte mantiene la equidistancia.

- Los porcentajes de «No contesta» ponen de manifiesto que, en general, el alumnado ha intentado contestar.

Un aspecto destacable es la existencia de un porcentaje importante de la muestra que tiene posiciones poco definidas, sobre todo si comparamos los porcentajes obtenidos en las cinco dicotomías con los de «Valoración global». Se podría interpretar que hay una cierta predisposición positiva, pero ante una visión negativa, el estudiante duda... y honestamente lo dice.

Pero también se puede valorar que las exigencias cognitivas de los planteamientos dicotómicos son muchas veces más complejas de lo que parecen. Ponemos mucho énfasis en el estudio de las leyes físiconaturales y dejamos de lado debates como los que implícitamente están en las dicotomías planteadas. ¿Qué visión trasmitimos de la ciencia en la educación obligatoria? ¿Hemos trasladado al aula alguna vez estos posicionamientos antagónicos? ¿Qué actividades concretas realizamos para que el alumnado pueda tener una posición, sea cual sea, en algunas de estas dicotomías? Como ya dijimos (Pro, 2003), aprender estos conocimientos no se realiza "por casualidad» ni "como consecuencia natural» de otros aprendizajes... y aunque a algunos les pueda sorprender, resultan complejos. Por ello, entendemos la equidistancia mencionada.

En cualquier caso, los porcentajes de posicionamientos positivos son mayores que los negativos en tres de las dicotomías y en la valoración global. Hay dos que se salen de esta clara tendencia: las relacionadas con las noticias de prensa. Resulta incauto ignorar que los medios de comunicación forman parte de la vida de los ciudadanos y que, querámoslo o no, en ellos se informa o desinforma sobre hechos y acontecimientos que tienen relación con los contenidos que enseñamos. Podemos discutir el interés por estos en estas edades o la idoneidad de su incorporación a las aulas por los problemas de comprensión lectora que, sin duda, existen, pero estos factores no son suficientes para pensar que nuestros alumnos son impermeables a lo que escuchan en sus casas, en los telediarios o en la calle. No podemos obviar esta circunstancia porque, si lo hacemos, estaremos aumentando la brecha entre lo que se hace dentro y fuera del aula.

Por último, como dijimos, creamos una variable - «Total dicotomía»- que fuera una combinación de las seis cuestiones planteadas. En total, tuvimos la información de 6.568 estudiantes; los restantes -248- no habían contestado a alguna de las cuestiones y lógicamente no los consideramos en este recuento. Como no pretendíamos obtener valores exactos sino solo apreciar las tendencias, fijamos unos intervalos y etiquetamos unas categorías en función de los valores de esta variable. En la tabla siguiente se recogen las frecuencias y porcentajes de cada una. 
Tabla 3 .

Resultados obtenidos en la variable «Total de dicotomías»

\begin{tabular}{|c|c|c|c|c|c|}
\hline Total dicotomía & Muy negativo & Negativa & Neutra & Positiva & Muy positiva \\
\hline Frecuencia & 159 & 1.047 & 3.360 & 1.884 & 128 \\
$\%$ & $2,3 \%$ & $15,3 \%$ & $49,2 \%$ & $27,6 \%$ & $1,9 \%$ \\
\hline
\end{tabular}

Puede verse que el valor de mayor frecuencia es la posición neutra (en torno a la mitad de la muestra), lo que a priori puede resultar un tanto decepcionante. Es cierto que las tendencias positivas superan sensiblemente las negativas pero, en estas edades, pensábamos que existía una cierta mitificación de las ciencias que se superponían a otros aspectos más criticables, es decir, esperábamos resultados más acordes con los obtenidos en la variable «Valoración global».

\section{Resultados de las posiciones ante las dicotomías en función del nivel educativo}

Los resultados obtenidos al cruzar los valores en cada dicotomía con el curso académico -sexto de EP y cuarto de ESO- se recogen en la siguiente tabla. Aparecen solo los porcentajes correspondientes a los dos grupos. Mantenemos el tramado para resaltar las opciones favorables, neutras y desfavorables. Hemos excluido los «No contesta» pues no aportan ninguna información relevante.

Tabla 4.

Resultados de las variables en función del nivel académico

\begin{tabular}{|c|c|c|c|c|c|c|c|c|c|c|c|c|}
\hline & \multirow{2}{*}{\multicolumn{2}{|c|}{$\begin{array}{c}\begin{array}{c}\text { Mejora en la } \\
\text { salud-Industria } \\
\text { militar }\end{array} \\
\text { Nivel educativo }\end{array}$}} & \multirow{2}{*}{\multicolumn{2}{|c|}{$\begin{array}{c}\begin{array}{c}\text { Estilo de vida- } \\
\text { Actitudes no } \\
\text { deseables }\end{array} \\
\text { Nivel educativo }\end{array}$}} & \multirow{2}{*}{\multicolumn{2}{|c|}{ 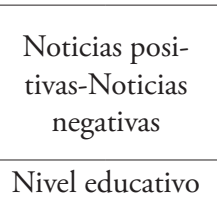 }} & \multirow{2}{*}{\multicolumn{2}{|c|}{\begin{tabular}{|c|}
$\begin{array}{c}\text { Credibilidad } \\
\text { (versión }\end{array}$ \\
favorable-versión \\
desfavorable)
\end{tabular}}} & \multirow{2}{*}{\multicolumn{2}{|c|}{\begin{tabular}{|c|}
$\begin{array}{c}\text { Avances sociales- } \\
\text { Problemas } \\
\text { sociales }\end{array}$ \\
Nivel educativo
\end{tabular}}} & \multirow{2}{*}{\multicolumn{2}{|c|}{$\begin{array}{c}\text { Valoración glo- } \\
\text { bal aportaciones } \\
\text { de ciencias }\end{array}$}} \\
\hline & & & & & & & & & & & & \\
\hline & 6. ${ }^{\circ} \mathrm{EP}$ & 4. ${ }^{\circ} \mathrm{ESO}$ & 6. ${ }^{\circ} \mathrm{EP}$ & 4. ${ }^{\circ} \mathrm{ESO}$ & 6. ${ }^{\circ} \mathrm{EP}$ & 4. ${ }^{\circ} \mathrm{ESO}$ & 6. ${ }^{\circ} \mathrm{EP}$ & $4 .^{\circ} \mathrm{ESO}$ & 6. ${ }^{\circ} \mathrm{EP}$ & 4. ${ }^{\circ} \mathrm{ESO}$ & 6. ${ }^{\circ} \mathrm{EP}$ & 4. ${ }^{\circ} \mathrm{ESO}$ \\
\hline $\begin{array}{l}\text { Solo las } \\
\text { positivas }\end{array}$ & $17,8 \%$ & $6,6 \%$ & $12,6 \%$ & $9,3 \%$ & & & $10,5 \%$ & $4,1 \%$ & $6,8 \%$ & $4,0 \%$ & $12,3 \%$ & $4,8 \%$ \\
\hline $\begin{array}{l}\text { Más las } \\
\text { positivas }\end{array}$ & $34,1 \%$ & $38,5 \%$ & $26,7 \%$ & $32,5 \%$ & $24,4 \%$ & $22,1 \%$ & & & $25,2 \%$ & $23,8 \%$ & $49,9 \%$ & $54,0 \%$ \\
\hline $\begin{array}{l}\text { Igual + } \\
\text { que - }\end{array}$ & $43,0 \%$ & $47,7 \%$ & $36,1 \%$ & $37,0 \%$ & $32,3 \%$ & $29,1 \%$ & $53,7 \%$ & $55,7 \%$ & $41,7 \%$ & $43,9 \%$ & & \\
\hline $\begin{array}{l}\mathrm{Ni}+\mathrm{ni} \\
\text { negativas }\end{array}$ & $1,1 \%$ & $2,2 \%$ & $2,0 \%$ & $2,3 \%$ & & & $9,7 \%$ & $9,1 \%$ & $4,0 \%$ & $7,0 \%$ & & \\
\hline No lo sé & & & & & $20,2 \%$ & $20,6 \%$ & $16,1 \%$ & $18,8 \%$ & & & $29,7 \%$ & $32,0 \%$ \\
\hline $\begin{array}{l}\text { Más las } \\
\text { negativas }\end{array}$ & $1,8 \%$ & $3,4 \%$ & $17,8 \%$ & $15,9 \%$ & $21,4 \%$ & $27,1 \%$ & & & $16,3 \%$ & $17 \%$ & $4,5 \%$ & $5,9 \%$ \\
\hline $\begin{array}{l}\text { Solo las } \\
\text { negativas }\end{array}$ & $0,8 \%$ & $0,7 \%$ & $3,6 \%$ & $2,1 \%$ & & & $8,3 \%$ & $10,8 \%$ & $4,3 \%$ & $2,6 \%$ & $1,4 \%$ & $1,1 \%$ \\
\hline
\end{tabular}

A la vista de los valores obtenidos podemos decir:

- En las dicotomías «Salud» y «Repercusiones sociales», hay un sensible descenso de las posiciones positivas y un aumento de las equidistantes con el nivel educativo (los de sexto de EP manifiestan opiniones más positivas).

- En relación con las «Noticias» y "Credibilidad», se mantiene la tendencia: posiciones más positivas en EP que en ESO; de hecho, las opciones negativas superan a las positivas en el grupo de cuartode la ESO. 
- En la dicotomía «Estilo de vida» existen las menores diferencias entre ambos colectivos.

- En «Valoración global» se mantiene la tendencia: visión más positiva de ambos colectivos -en relación con las dicotomías- y posicionamientos más positivos en sexto de EP.

Hemos calculado y etiquetado también la variable «Total dicotomía» y los valores se recogen en la siguiente tabla. Aparecen las frecuencias y los porcentajes correspondientes (excluimos los que no disponemos de la información de todas las dicotomías).

Tabla 5 .

Resultados de la variable «Total dicotomía» en función del nivel educativo

\begin{tabular}{|c|c|c|c|c|c|c|c|c|c|c|}
\hline \multirow{2}{*}{$\begin{array}{c}\text { Total } \\
\text { dicotomía }\end{array}$} & \multicolumn{2}{|c|}{ Muy negativo } & \multicolumn{2}{c|}{ Negativa } & \multicolumn{2}{c|}{ Neutra } & \multicolumn{2}{c|}{ Positiva } & \multicolumn{2}{c|}{ Muy positiva } \\
\cline { 2 - 12 } & $6 .{ }^{\circ} \mathrm{EP}$ & $4 .{ }^{\circ} \mathrm{ESO}$ & $6 .{ }^{\circ} \mathrm{EP}$ & $4 .^{\circ} \mathrm{ESO}$ & $6 .{ }^{\circ} \mathrm{EP}$ & $4 .{ }^{\circ} \mathrm{ESO}$ & $6 .{ }^{\circ} \mathrm{EP}$ & $4 .{ }^{\circ} \mathrm{ESO}$ & $6 .{ }^{\circ} \mathrm{EP}$ & $4 .{ }^{\circ} \mathrm{ESO}$ \\
\hline $\begin{array}{c}\text { Frecuencia } \\
\%\end{array}$ & 73 & 86 & 540 & 507 & 1.917 & 1.443 & 1.106 & 778 & 108 & 20 \\
\hline
\end{tabular}

Como puede verse, la categoría que recoge un porcentaje más alto de alumnos en ambos niveles educativos es la «Neutra». No obstante, en los dos grupos las categorías «Positiva»-«Muy positiva» superan a las «Negativa»-«Muy Negativa».

Por otro lado, se aprecian diferencias entre los grupos: las percepciones son más positivas al terminar la educación primaria que al terminar la educación secundaria.

\section{Resultados de las posiciones ante las dicotomías en función del género}

Los resultados obtenidos al cruzar los datos con la variable género se recogen en la siguiente tabla; aparecen solo los porcentajes de cada grupo ( $\mathrm{H}$ : hombre; $\mathrm{M}$ : mujer); hemos excluido los «No contesta».

Tabla 6.

Resultados de las variables en función del género

\begin{tabular}{|c|c|c|c|c|c|c|c|c|c|c|c|c|}
\hline & \multirow{2}{*}{\multicolumn{2}{|c|}{$\begin{array}{c}\text { Mejora en la } \\
\text { salud-Industria } \\
\text { militar }\end{array}$}} & \multicolumn{2}{|c|}{$\begin{array}{l}\text { Estilo de vida- } \\
\text { Actitudes no } \\
\text { deseables }\end{array}$} & \multicolumn{2}{|c|}{$\begin{array}{l}\text { Noticias } \\
\text { positivas- } \\
\text { Noticias } \\
\text { negativa }\end{array}$} & \multicolumn{2}{|c|}{$\begin{array}{l}\text { Credibilidad } \\
\text { (versión } \\
\text { favorable- } \\
\text { versión } \\
\text { desfavorable) }\end{array}$} & \multicolumn{2}{|c|}{$\begin{array}{l}\text { Avances } \\
\text { sociales- } \\
\text { Problemas } \\
\text { sociales }\end{array}$} & \multicolumn{2}{|c|}{$\begin{array}{l}\text { Valoración } \\
\text { global } \\
\text { aportaciones } \\
\text { de ciencias }\end{array}$} \\
\hline & & & Gé & ero & Gé & ero & Gé & ero & Gér & ero & Gér & hero \\
\hline & $\mathrm{H}$ & $M$ & $\mathrm{H}$ & M & $\mathrm{H}$ & $M$ & $\mathrm{H}$ & M & $\mathrm{H}$ & $M$ & $\mathrm{H}$ & $\mathrm{M}$ \\
\hline $\begin{array}{l}\text { Solo las } \\
\text { positivas }\end{array}$ & $12,7 \%$ & $13,4 \%$ & $13,7 \%$ & $8,6 \%$ & & & $9,1 \%$ & $6,2 \%$ & $6,4 \%$ & $4,7 \%$ & $10,5 \%$ & $7,5 \%$ \\
\hline $\begin{array}{l}\text { Más las } \\
\text { positivas }\end{array}$ & $33,3 \%$ & $38,9 \%$ & $29,2 \%$ & $29,3 \%$ & $26,8 \%$ & $20,1 \%$ & & & $24,1 \%$ & $25,1 \%$ & $53,9 \%$ & $49,4 \%$ \\
\hline Igual + que - & $46,5 \%$ & $43,6 \%$ & $34,4 \%$ & $38,6 \%$ & $28,9 \%$ & $33,0 \%$ & $52,7 \%$ & $56,6 \%$ & $41,8 \%$ & $43,7 \%$ & & \\
\hline $\begin{array}{l}\mathrm{Ni}+\text { ni } \\
\text { negativas }\end{array}$ & $2,3 \%$ & $0,8 \%$ & $2,9 \%$ & $3,0 \%$ & & & $11,1 \%$ & $7,7 \%$ & $6,1 \%$ & $4,4 \%$ & & \\
\hline No lo sé & & & & & $17,3 \%$ & $23,6 \%$ & $15,4 \%$ & $19,3 \%$ & & & $26,6 \%$ & $35,3 \%$ \\
\hline $\begin{array}{l}\text { Más las } \\
\text { negativas }\end{array}$ & $3,0 \%$ & $1,9 \%$ & $16,2 \%$ & $17,8 \%$ & $25,7 \%$ & $21,9 \%$ & & & $16,2 \%$ & $17,1 \%$ & $5,0 \%$ & $4,7 \%$ \\
\hline $\begin{array}{l}\text { Solo las } \\
\text { negativas }\end{array}$ & $1,0 \%$ & $0,6 \%$ & $2,9 \%$ & $3,0 \%$ & & & $10,1 \%$ & $8,7 \%$ & $3,9 \%$ & $3,3 \%$ & $1,6 \%$ & $1,0 \%$ \\
\hline
\end{tabular}


A la vista de los valores obtenidos podemos decir:

- Persisten las características ya apuntadas, tanto en los chicos como en las chicas: predominio abrumador de las posiciones equidistantes en todas las dicotomías y más positivas que neutras o negativas en «Valoración global»...

- En la dicotomía «Salud», las posiciones son sensiblemente más positivas en las chicas; en las dicotomías "Estilo de vida» y "Noticias», son sensiblemente más positivas en los chicos; en las restantes, las diferencias no resultan relevantes.

- En la «Valoración global» hay posiciones más positivas respecto a las ciencias en los chicos.

También hemos utilizado la variable "Total dicotomía», cuyos valores se recogen en la siguiente tabla. Aparecen las frecuencias y los porcentajes de cada uno de los intervalos (excluimos los que no tenemos todos los datos).

Tabla 7.

Resultados de la variable «Total dicotomía» en función del género

\begin{tabular}{|c|c|c|c|c|c|c|c|c|c|c|}
\hline \multirow{2}{*}{ Total dicotomía } & \multicolumn{2}{|c|}{ Muy negativa } & \multicolumn{2}{c|}{ Negativa } & \multicolumn{2}{c|}{ Neutra } & \multicolumn{2}{c|}{ Positiva } & \multicolumn{2}{c|}{ Muy positiva } \\
\cline { 2 - 11 } & $\mathrm{H}$ & $\mathrm{M}$ & $\mathrm{H}$ & $\mathrm{M}$ & $\mathrm{H}$ & $\mathrm{M}$ & $\mathrm{H}$ & $\mathrm{M}$ & $\mathrm{H}$ & $\mathrm{M}$ \\
\hline $\begin{array}{c}\text { Frecuencia } \\
\%\end{array}$ & 73 & 84 & 537 & 506 & 1677 & 1665 & 990 & 887 & 68 & 55 \\
& $2,1 \%$ & $2,5 \%$ & $15,5 \%$ & $15,3 \%$ & $48,3 \%$ & $50,3 \%$ & $28,5 \%$ & $26,8 \%$ & $2,0 \%$ & $1,7 \%$ \\
\hline
\end{tabular}

Nuevamente la categoría que recoge un porcentaje más alto, tanto en los chicos como en las chicas, es la «Neutra» (en torno a la mitad de cada grupo). Y en ambos colectivos las categorías «Positiva»«Muy positiva» superan a las «Negativa»-«Muy Negativa».

Por otro lado, no se aprecian grandes diferencias entre los grupos. Aunque parece existir a una visión sensiblemente más positiva por los chicos, la diferencia no es tan determinante como en el cruce respecto al nivel educativo.

\section{Resultados de las posiciones ante las dicotomías en función del tipo de centro}

Los resultados obtenidos al cruzar los datos con la variable tipo de centro - público o privado- se recogen en la siguiente tabla 8; como en los cruces anteriores, aparecen solo los porcentajes de cada grupo.

Tabla 8.

Resultados de las variables en función del tipo de centro

\begin{tabular}{|c|c|c|c|c|c|c|c|c|c|c|c|c|}
\hline & \multirow{2}{*}{\multicolumn{2}{|c|}{$\begin{array}{c}\text { Mejora en la } \\
\text { salud-Industria } \\
\text { militar }\end{array}$}} & \multicolumn{2}{|c|}{$\begin{array}{l}\text { Estilo de vida- } \\
\text { Actitudes no } \\
\text { deseables }\end{array}$} & \multicolumn{2}{|c|}{$\begin{array}{l}\text { Noticias } \\
\text { positivas- } \\
\text { Noticias } \\
\text { negativas }\end{array}$} & \multicolumn{2}{|c|}{$\begin{array}{l}\text { Credibilidad } \\
\text { (versión } \\
\text { favorable- } \\
\text { versión } \\
\text { desfavorable) }\end{array}$} & \multicolumn{2}{|c|}{$\begin{array}{l}\text { Avances } \\
\text { sociales- } \\
\text { Problemas } \\
\text { sociales }\end{array}$} & \multicolumn{2}{|c|}{$\begin{array}{c}\text { Valoración } \\
\text { global } \\
\text { aportaciones de } \\
\text { ciencias }\end{array}$} \\
\hline & & & \multicolumn{2}{|c|}{ Tipo de centro } & \multicolumn{2}{|c|}{ Tipo de centro } & \multicolumn{2}{|c|}{ Tipo de centro } & \multicolumn{2}{|c|}{ Tipo de centro } & \multicolumn{2}{|c|}{ Tipo de centro } \\
\hline & Público & Privado & Público & Privado & Público & Privado & Público & Privado & Público & Privado & Público & Privado \\
\hline $\begin{array}{l}\text { Solo las } \\
\text { positivas }\end{array}$ & $13,7 \%$ & $11,6 \%$ & $11,4 \%$ & $10,7 \%$ & & & $7,5 \%$ & $8,3 \%$ & $5,7 \%$ & $5,7 \%$ & $9,0 \%$ & $9,3 \%$ \\
\hline $\begin{array}{l}\text { Más las } \\
\text { positivas }\end{array}$ & $34,7 \%$ & $38,6 \%$ & $28,7 \%$ & $30,2 \%$ & $23,4 \%$ & $23,5 \%$ & & & $24,2 \%$ & $25,5 \%$ & $50,0 \%$ & $54,8 \%$ \\
\hline
\end{tabular}




\begin{tabular}{|c|c|c|c|c|c|c|c|c|c|c|c|c|}
\hline & \multirow{2}{*}{\multicolumn{2}{|c|}{$\begin{array}{c}\text { Mejora en la } \\
\text { salud-Industria } \\
\text { militar }\end{array}$}} & \multicolumn{2}{|c|}{$\begin{array}{l}\text { Estilo de vida- } \\
\text { Actitudes no } \\
\text { deseables }\end{array}$} & \multicolumn{2}{|c|}{$\begin{array}{l}\text { Noticias } \\
\text { positivas- } \\
\text { Noticias } \\
\text { negativas }\end{array}$} & \multicolumn{2}{|c|}{$\begin{array}{l}\text { Credibilidad } \\
\text { (versión } \\
\text { favorable- } \\
\text { versión } \\
\text { desfavorable) }\end{array}$} & \multicolumn{2}{|c|}{$\begin{array}{l}\text { Avances } \\
\text { sociales- } \\
\text { Problemas } \\
\text { sociales }\end{array}$} & \multicolumn{2}{|c|}{$\begin{array}{c}\text { Valoración } \\
\text { global } \\
\text { aportaciones de } \\
\text { ciencias }\end{array}$} \\
\hline & & & Tipo d & centro & Tipo a & centro & Tipo d & centro & Tipod & centro & Tipo a & centro \\
\hline & Público & Privado & Público & Privado & Público & Privado & Público & Privado & Público & Privado & Público & Privado \\
\hline $\begin{array}{l}\text { Igual + } \\
\text { que - }\end{array}$ & $45,2 \%$ & $44,7 \%$ & $36,0 \%$ & $37,5 \%$ & $30,9 \%$ & $30,9 \%$ & $54,6 \%$ & $54,5 \%$ & $41,9 \%$ & $44,1 \%$ & & \\
\hline $\begin{array}{l}\mathrm{Ni}+\text { ni } \\
\text { negativas }\end{array}$ & $1,9 \%$ & $1 \%$ & $2,4 \%$ & $1,5 \%$ & & & $9,5 \%$ & $9,4 \%$ & $5,8 \%$ & $4,1 \%$ & & \\
\hline No lo sé & & & & & $20,8 \%$ & $19,4 \%$ & $16,8 \%$ & $18,2 \%$ & & & $31,9 \%$ & $28,5 \%$ \\
\hline $\begin{array}{l}\text { Más las } \\
\text { negativas }\end{array}$ & $2,5 \%$ & $2,5 \%$ & $17,4 \%$ & $16,2 \%$ & $23,2 \%$ & $25,2 \%$ & & & $16,8 \%$ & $16,0 \%$ & $5,2 \%$ & $5,1 \%$ \\
\hline $\begin{array}{l}\text { Solo las } \\
\text { negativas }\end{array}$ & $0,8 \%$ & $0,7 \%$ & $2,9 \%$ & $3,1 \%$ & & & $9,9 \%$ & $8,4 \%$ & $3,6 \%$ & $3,7 \%$ & $1,5 \%$ & $0,9 \%$ \\
\hline
\end{tabular}

A la vista de los valores obtenidos podemos decir:

- Persisten las características ya apuntadas, tanto en los centros públicos como privados: posiciones equidistantes en las dicotomías y más positivas que neutras o negativas en la «Valoración global».

- En la dicotomía «Salud», parece que los centros públicos tienen percepciones más positivas; en las demás, se observa un equilibrio en las visiones de ambos colectivos.

- En «Valoración global» hay posiciones más positivas respecto a las ciencias en los privados.

También hemos utilizado la variable «Total dicotomías», cuyos valores se recogen en la siguiente tabla. Aparecen las frecuencias y los porcentajes correspondientes.

Tabla 9.

Resultados de la variable «Total dicotomía» en función del tipo de centro

\begin{tabular}{|c|c|c|c|c|c|c|c|c|c|c|}
\hline \multirow{2}{*}{ Total dicotomía } & \multicolumn{2}{|c|}{ Muy negativa } & \multicolumn{2}{c|}{ Negativa } & \multicolumn{2}{c|}{ Neutra } & \multicolumn{2}{c|}{ Positiva } & \multicolumn{2}{c|}{ Muy positiva } \\
\cline { 2 - 10 } & Público & Privado & Público & Privado & Público & Privado & Público & Privado & Público & Privado \\
\hline Frecuencia & 104 & 55 & 719 & 328 & 2.200 & 1.160 & 1.218 & 666 & 88 & 40 \\
$\%$ & $2,3 \%$ & $2,4 \%$ & $15,9 \%$ & $14,2 \%$ & $48,7 \%$ & $50,1 \%$ & $27,0 \%$ & $28,8 \%$ & $1,9 \%$ & $1,7 \%$ \\
\hline
\end{tabular}

Una vez más la categoría que recoge un porcentaje más alto de centros públicos y centros privados es la «Neutra». No obstante, en los dos grupos las categorías «Positiva»-«Muy positiva» superan a las «Negativa»-«Muy Negativa». Por otro lado, no se aprecian diferencias importantes entre estos colectivos.

\section{CONCLUSIONES}

Este trabajo forma parte de un estudio demoscópico más amplio sobre las actitudes de los niños y adolescentes en España. En particular, en este nos hemos centrado en qué posicionamientos tienen estos colectivos cuando se plantean dicotomías entre aportaciones positivas y cautelas de los descubrimientos científicos en la vida cotidiana.

Revisamos algunas aportaciones sobre las actitudes detectando que existen muchas acepciones (hacia la ciencia y sus descubrimientos, hacia los científicos y su trabajo, en una actividad científica, hacia 
las materias de ciencias...) y que parece existir una cierta dependencia de variables escolares (por ejemplo, del nivel educativo) y sociales (por ejemplo, del género, del contexto, de las comunidades autónomas, etc.). No obstante, percibimos que muchas veces los resultados se entremezclan, no dejando claro los que se refieren a las actitudes hacia las ciencias y sus descubrimientos, que constituyen el objetivo central de este trabajo.

Luego hemos analizado su presencia en el currículo oficial que cursaron los alumnos objeto de nuestro estudio. Con ello, hemos constatado que, en la educación obligatoria, se han debido abordar las actitudes como contenidos objeto de enseñanza (obviamente, no sabemos ni cómo se ha hecho ni la importancia asignada) y, por tanto, que la visión que tienen sobre la ciencia y sus descubrimientos no debería ser «fruto exclusivo» del contexto existente fuera de la escuela (familia, conversaciones con amigos, publicidad, noticias, etc.).

Hemos seleccionado una muestra por conglomerados a partir de los datos oficiales, en función de las comunidades autónomas, nivel educativo y tipo de centro. Se ha diseñado y aplicado un cuestionario (PANA); en este trabajo solo hemos ofrecido la información de seis de las cuestiones planteadas: cinco dicotomías y una valoración global. Las primeras se han referido a la «Salud» (aportaciones a la medicina vs. industria militar), "Estilo de vida» (mejora de estilo de vida frente a actitudes no deseables), «Noticias» (noticias positivas frente a catástrofes, calamidades...), «Credibilidad» (versiones contradictorias de la misma noticia) y «Repercusiones sociales» (avances, nuevas profesiones, etc. frente a problemas sociales). Todas ellas eran de opción múltiple.

Los resultados obtenidos en relación con el PP1 ponen de manifiesto que, cuando se plantean las dicotomías, predominan las posturas equidistantes. Esta equidistancia no se ha percibido en los trabajos revisados, ya que no se contraponían los efectos positivos y negativos; de hecho, en el proyecto ROSE (en los cuestionarios D -los cambios ambientales- y, sobre todo, G - mis opiniones sobre ciencia y tecnología-, que son los más relacionados con este trabajo), el alumnado debe posicionarse sobre un conjunto de afirmaciones, abrumadoramente positivas ¿Se obtendrían los mismos resultados ante situaciones dicotómicas? Creemos que no. De hecho, en nuestra experiencia, hemos detectado diferencias entre los resultados dicotómicos y los obtenidos en la «Valoración global» (cuya formulación está más "en la línea» de afirmaciones procientíficas del proyecto mencionado). Ante esta situación, ¿no sería conveniente completar la información con cuestiones de tipo dicotómico para conocer qué es lo que cree realmente el alumnado?

Los posicionamientos equidistantes, en nuestro caso, pueden ser debido a la dificultad intrínseca de lo que se plantea, pero sobre todo se detecta que este tipo de debates no se han abordado en las clases durante la educación obligatoria. No obstante, existe una cierta predisposición positiva (frecuencias más altas que las negativas) -en menor grado, quizás, que la encontrada por Vázquez y Manasero (2009) - que, en cualquier caso, debería ser aprovechada.

Por otro lado, en relación con el PP2, hemos podido observar que las posiciones son más positivas en educación primaria que en la ESO. Aunque este resultado está en la línea de otros estudios -como vimos en el primer apartado- no deja de ser preocupante. En nuestro caso, además, hemos podido identificar los posicionamientos de los alumnos al terminar la educación primaria, lo que supone un referente importante, ya que no conocemos que se haya identificado en otros estudios con una muestra del tamaño que hemos estudiado. Incluso hemos detectado que las mayores diferencias se dan en las cuestiones en las que intervienen los medios de comunicación, dato que abre un campo de indagación -curiosamente bastante alejado de lo que habitualmente se trabaja en las clases de ciencias- que no había sido contemplado en los trabajos revisados.

En cuanto al género, se observa una cierta tendencia a que la visión sea «un poco» más positiva en los chicos, pero desde luego las diferencias detectadas están alejadas de algunos de los resultados de otros trabajos que recogimos en el primer apartado. Tampoco parecían claras las diferencias en el traba- 
jo de Vázquez y Manasero (2009) cuando debían posicionarse en contra de algunas de las afirmaciones que se les planteaban. Incluso, en nuestro estudio, hay un ámbito -el de «Salud»- en el que los resultados de las chicas responden a una visión más positiva de las ciencias. Creemos que la dependencia del género exige más estudios.

En cuanto al tipo de centro, no hemos encontrado diferencias destacables a favor de ninguno de ellos. A la vista de los resultados, en nuestro contexto educativo probablemente hay factores que inciden de forma más relevante en los posicionamientos; algunos los hemos detectado y otros habrá que seguir buscándolos.

Por último, quisiéramos compartir una reflexión. Si los diseñadores curriculares o los profesores creen que este tipo de contenidos o estas dicotomías no son útiles para la formación de un ciudadano, nosotros no lo compartimos, pero, por coherencia, deberían ser excluidos de los programas oficiales y de las programaciones. Si, por lo contrario, creen en su necesidad y utilidad, los resultados muestran que hay que cambiar algo de lo que estamos haciendo porque el estudiante, como hemos podido apreciar, no tiene los referentes para elegir y tomar decisiones con fundamento. No podemos seguir engañándonos con declaraciones e intenciones en los papeles -insistir en la importancia y necesidad de trabajar las actitudes en las clases de ciencias- cuando estas no tienen que ver con lo que realmente pensamos y, sobre todo, hacemos en las aulas.

\section{REFERENCIAS BIBLIOGRÁFICAS}

Acevedo, J.A. (2005). Proyecto ROSE: Relevancia de la Educación Científica. Revista Eureka sobre Enseñanza y Divulgación de las Ciencias, 2 (3), pp. 440-447.

Allport, G.W. (1935). Attitudes. En C. Murchinson (dir.). Handbook of Social Psychology. Worcester, Clark University Press, pp. 798-884.

Barmby, P.; Kind, P.M. y Jones, K. (2008). Examining changing attitudes in Secondary School Science. Internacional Journal of Science Education, 30(8), pp. 1075-1093.

European Comision (2004). Europe needs more scientifics. Luxembourg: Office for Oficial Publications of European Communities.

George, R. (2006). A cross-domain análisis of change in students' attitudes toward science and attitudes about the utility of science. Internacional Journal of Science Education, 28 (6), pp. 571-589.

Gibson, H.L. y Chase C. (2002). Longitudinal impact of an inquiry-based Science Program on Middle School students' attitudes toward Science. Science Education, 86 (5), pp. 693-705.

GiL, J. (2012). Actitudes del alumnado español hacia las ciencias en la evaluación PISA 2006. Enseñanza de las Ciencias, 30 (2), pp. 131-152.

Jenkins, E.W. y Pell, R.G. (2006). The relevance of Science Education Project (ROSE) in England: a summary of findings. Leeds: University of Leeds.

Kind, P.; Jones, K. y Barmby, P. (2007). Developing attitudes toward science measures. Internacional Journal of Science Education, 29 (7), pp. 871-893.

Lindahl, B. (2005, septiembre). A Longitudinal study about attitudes to science. Comunicación presentada en 4th ESERA Conference. Barcelona.

López, L.; Pro, A. y Saura, O. (2012, junio). ¿Cambian todas las actitudes con una propuesta innovadora? Comunicación presentada al I Simposium Internacional de Enseñanza de las Ciencias ISIEC 2012. Vigo.

Marbá-Tallada, A. y Márquez, C. (2010). ¿Qué opinan los estudiantes de las clases de Ciencias? Un estudio trasversal de sexto de Primaria a Cuarto de la ESO. Enseñanza de las Ciencias, 28(1), pp. 19-30. 
MEC (1992a). Real Decreto por el que se establecen las enseñanzas mínimas de la Educación Primaria. MEC (1992b). Real Decreto por el que se establecen las enseñanzas mínimas correspondientes a la Educación Secundaria Obligatoria.

Murphy, C. y Beggs, J. (2003). Children perceptions of school science. School Science Review, 84 (308), pp. 109-116.

Osborne, J.; Simon, S. y Collins, S. (2003). Attitudes toward science: a review of the literature and its implications. Internacional Journal of Science Education, 23 (5), pp. 448-467.

Pell, T. y Jarvis, T. (2001). Developing attitude to science scales for use with children of ages from five to eleven years. International Journal of Science Education, 23 (8), pp. 847- 862.

Pérez, A. (2013). Actitudes hacia la Ciencia en Primaria y Secundaria. Tesis doctoral. Universidad de Murcia.

Pérez, A. y Pro, A. (2005). Evaluación nacional de actitudes y valores hacia la ciencia en entornos educativos. Madrid: FECYT.

Pérez, A. y Pro, A. (2013). Estudio demoscópico de lo que sienten y piensan los niños y adolescentes sobre la enseñanza formal de las ciencias. En V. Mellado, L. Blanco, A. Borrachero y J. Cárdenas. Las Emociones en la Enseñanza y el Aprendizaje de las Ciencias y las Matemáticas. Volumen II. Badajoz: Grupo de investigación DEPROFE, pp. 493-518.

Pro, A. (2003). La construcción del conocimiento científico y los contenidos de ciencias. En M.P. Jiménez (coord.). Enseñar Ciencias. Barcelona. Graó, pp. 33-54.

Pro, A. (2006). Perfil de la reforma LOGSE y perfil de uso. Los fundamentos de los proyectos curriculares de Física y Química en centros de secundaria. Enseñanza de las Ciencias, 24(2), pp. 337-356.

Pro, A. (2007). Los contenidos e los proyectos curriculares de Física y Química en centros de secundaria en la implantación de la reforma LOGSE. Enseñanza de las Ciencias, 25 (3), pp. 367-386.

Pro, A. y Pérez, A. (2013a). Cuestionario del Bloque Dicotomías. Disponible en línea: http://www. um.es/pana/pana/Dicotomias_files/Cuestionario\%20Bloque\%20Dicotomi\%CC\%81as.pdf. (Última consulta, 26 de marzo de 2013).

Pro, A. y Pérez, A. (2013b). Instrucciones a los coordinadores. Disponible en línea: http://www. um.es/pana/pana/Dicotomias_files/Instrucciones\%20a\%20los\%20coordinadores\%20responsables.pdf. (Última consulta, 26 de marzo de 2013).

Pro, A.; Pérez, A. y Tárraga, P. (2009). ¿Científico? Sí, pero... Opinión de los escolares españoles sobre los científicos y su trabajo. Enseñanza de las Ciencias, Número extra VIII, Congreso Internacional sobre Investigación en Didáctica de las Ciencias, pp. 3649-3656.

Pro, A.; Sánchez, G. y Valcárcel, M.V. (2008). Análisis de los libros de texto de Física y Química en el contexto de la Reforma LOGSE. Enseñanza de las Ciencias, 26 (2), pp. 189-206.

Schreiner, C. y Sjøberg, S. (2004). Sowing the seeds of ROSE. Background, Rationale, Questionnaire Development and Data Collection for ROSE (The Relevance of Science Education) - A comparative study of students' views of science and science education. Acta Didactica 4/2004. Oslo: Dept. of Teacher Education and School Development. Disponible en línea: http://www.uv.uio. no/ils/english/research/projects/rose/publications/sowing-rose.pdf. (Última consulta, 15 agosto de 2012).

SJøberg, S. (2000). Science and Scientists The SAS-study. Acta Didactica 1/2000. Oslo: Dept. of Teacher Education and School Development. Disponible en línea: http://folk. uio.no/sveinsj/SASweb. htm. (Última consulta, 15 de agosto de 2012).

Sjøberg, S. y Schreiner, C. (2005, septiembre). Perceptions and images of science and science education. Some results from the ROSE Project in 32 countries. Comunicación presentada en 4 th ESERA Conference. Barcelona. 
Sjøberg, S. y Schreiner, C. (2007). Perceptions and images of science and science education. En M. Claessens (ed.). Communicating European Research 2005. Dordrecht: Springer, pp. 151-158.

Sjøberg, S. y Schreiner, C. (2010). The ROSE Project. An overview and key findings. University of Oslo. Disponible en línea: http://eacea.ec.europa.eu/education/eurydice/ comparative study on Science and Mathematics education. (Última consulta, 15 de agosto de 2012).

Speering, W. y Rennie, L.J. (1996). Students' perceptions about science: the impact of transition from primary to secondary school. Research in Science Education, 26 (3), pp. 283-298.

VÁzquez, A.; Acevedo, J.A. y Manassero, M.A. (2005). Más allá de una enseñanza de las ciencias para científicos: hacia una educación científica humanística. Revista Electrónica de Enseñanza de las Ciencias, 4(2). Disponible en línea: http://www.saum.uvigo.es/reec/volumenes/volumen4/ART5_ Vol4_N2.pdf. (Última consulta, 15 de agosto de 2012).

VÁzquez, A. y Manassero, M.A. (2007a). La relevancia de la educación científica. Palma de Mallorca: Servei de Publicacions de la Universitat de les Illes Balears.

VÁzquez, A. y Manassero, M.A. (2007b). En defensa de las actitudes y emociones en la educación científica (I): evidencias y argumentos generales. Revista Eureka sobre Enseñanza y Divulgación de las Ciencias, 4 (2), pp. 247-271.

VÁzquez, A. y Manassero, M.A. (2007c). En defensa de las actitudes y emociones en la educación científica (II): evidencias empíricas derivadas de la investigación. Revista Eureka sobre Enseñanza y Divulgación de las Ciencias, 4 (3), pp. 417-441.

VÁzquez, A. y Manassero, M.A. (2008). Las actitudes y la elección de ciencias en la educación obligatoria. Revista Eureka sobre Enseñanza y Divulgación de las Ciencias, 5 (3), pp. 274-292.

VÁzquez, A. y Manassero, M.A. (2009). La relevancia de la educación científica: actitudes y valores de los estudiantes relacionados con la Ciencia y la Tecnología. Enseñanza de las Ciencias, 27 (1), pp. 33-48. 


\section{ANEXO 1 \\ Datos de la muestra}

Datos de los porcentajes de los estratos, número de centros y número de alumnos por comunidad autónoma en educación primaria

\begin{tabular}{|c|c|c|c|c|c|c|c|c|c|}
\hline \multirow{3}{*}{ Comunidad autónoma } & \multicolumn{9}{|c|}{ Educación primaria } \\
\hline & \multicolumn{3}{|c|}{ Centros públicos } & \multicolumn{3}{|c|}{ Centros privados } & \multicolumn{3}{|c|}{ Total } \\
\hline & $\%$ & \begin{tabular}{|c|} 
N.o \\
centros
\end{tabular} & $\begin{array}{c}\text { N.o } \\
\text { alumnos }\end{array}$ & $\%$ & $\begin{array}{c}\text { N. }{ }^{\circ} \\
\text { centros }\end{array}$ & $\begin{array}{c}\text { N.o } \\
\text { alumnos }\end{array}$ & $\%$ & \begin{tabular}{|c|} 
N.o \\
centros
\end{tabular} & $\begin{array}{c}\text { N.o } \\
\text { alumnos }\end{array}$ \\
\hline Andalucía & 9,3 & 40 & 632 & 3,0 & 13 & 207 & 12,4 & 53 & 839 \\
\hline Aragón & 0,9 & 4 & 61 & 0,6 & 2 & 41 & 1,4 & 6 & 102 \\
\hline Asturias & 0,7 & 3 & 48 & 0,3 & 1 & 35 & 1,0 & 4 & 83 \\
\hline Baleares & 0,8 & 3 & 54 & 0,5 & 2 & 34 & 1,3 & 5 & 88 \\
\hline Canarias & 2,1 & 9 & 143 & 0,7 & 3 & 48 & 2,8 & 12 & 191 \\
\hline Cantabria & 0,4 & 2 & 27 & 0,2 & 1 & 15 & 0,6 & 3 & 42 \\
\hline Castilla y León & 1,8 & 8 & 123 & 1,0 & 4 & 65 & 2,8 & 12 & 188 \\
\hline Castilla-La Mancha & 2,3 & 10 & 156 & 0,5 & 2 & 33 & 2,7 & 12 & 189 \\
\hline Cataluña & 4,9 & 21 & 333 & 3,3 & 14 & 228 & 8,2 & 35 & 561 \\
\hline Comunidad Valenciana & 4,1 & 17 & 280 & 2,1 & 9 & 139 & 6,1 & 26 & 419 \\
\hline Extremadura & 1,3 & 6 & 88 & 0,3 & 2 & 24 & 1,6 & 8 & 112 \\
\hline Galicia & 2,1 & 9 & 143 & 0,9 & 4 & 60 & 2,9 & 13 & 203 \\
\hline Madrid (Comunidad de) & 4,0 & 17 & 273 & 3,4 & 15 & 232 & 7,4 & 32 & 505 \\
\hline Murcia (Región de) & 1,5 & 6 & 102 & 0,5 & 2 & 34 & 2,0 & 8 & 136 \\
\hline Navarra (Com. Foral de) & 0,5 & 2 & 31 & 0,3 & 1 & 20 & 0,8 & 3 & 51 \\
\hline País Vasco & 1,0 & 5 & 68 & 1,2 & 5 & 82 & 2,2 & 10 & 150 \\
\hline La Rioja & 0,2 & 1 & 24 & 0,1 & 0 & 0 & 0,3 & 1 & 24 \\
\hline Ceuta y Melilla & 0,1 & 1 & 12 & 0,1 & 0 & 0 & 0,2 & 1 & 12 \\
\hline Total & $38,00 \%$ & 165 & 2.591 & $18,95 \%$ & 80 & 1.304 & $56,95 \%$ & 244 & 3.895 \\
\hline
\end{tabular}

Datos de los porcentajes de los estratos, número de centros y número de alumnos por comunidad autónoma en Educación Secundaria

\begin{tabular}{|c|c|c|c|c|c|c|c|c|c|}
\hline \multirow{3}{*}{ Comunidad autónoma } & \multicolumn{9}{|c|}{ Educación Secundaria } \\
\hline & \multicolumn{3}{|c|}{ Centros públicos } & \multicolumn{3}{|c|}{ Centros privados } & \multicolumn{3}{|c|}{ Total } \\
\hline & $\%$ & $\begin{array}{c}\text { N.o } \\
\text { centros }\end{array}$ & $\begin{array}{c}\text { N.o } \\
\text { alumnos }\end{array}$ & $\%$ & $\begin{array}{c}\text { N.o } \\
\text { centros }\end{array}$ & $\begin{array}{c}\text { N.o } \\
\text { alumnos }\end{array}$ & $\%$ & $\begin{array}{c}\text { N.o } \\
\text { centros }\end{array}$ & $\begin{array}{c}\text { N.o } \\
\text { alumnos }\end{array}$ \\
\hline Andalucía & 6,9 & 30 & 470 & 2,4 & 9 & 163 & 9,3 & 39 & 633 \\
\hline Aragón & 0,7 & 3 & 48 & 0,4 & 2 & 28 & 1,1 & 5 & 76 \\
\hline Asturias & 0,5 & 3 & 20 & 0,3 & 1 & 24 & 0,8 & 4 & 44 \\
\hline Baleares & 0,5 & 2 & 34 & 0,4 & 2 & 27 & 0,9 & 4 & 61 \\
\hline Canarias & 1,7 & 7 & 116 & 0,5 & 2 & 32 & 2,2 & 9 & 148 \\
\hline Cantabria & 0,3 & 1 & 20 & 0,2 & 1 & 14 & 0,5 & 2 & 34 \\
\hline Castilla y León & 1,5 & 7 & 102 & 0,9 & 4 & 58 & 2,4 & 11 & 160 \\
\hline Castilla-La Mancha & 1,6 & 7 & 113 & 0,4 & 2 & 27 & 2,0 & 9 & 140 \\
\hline Cataluña & 3,4 & 14 & 231 & 2,6 & 9 & 177 & 6,0 & 23 & 408 \\
\hline Comunidad Valenciana & 3,0 & 13 & 204 & 1,5 & 7 & 102 & 4,5 & 20 & 306 \\
\hline Extremadura & 1,1 & 5 & 72 & 0,3 & 2 & 21 & 1,3 & 7 & 93 \\
\hline Galicia & 1,8 & 8 & 124 & 0,7 & 3 & 48 & 2,5 & 11 & 172 \\
\hline Madrid (Comunidad de) & 3,0 & 13 & 204 & 2,5 & 9 & 172 & 5,5 & 22 & 376 \\
\hline Murcia (Región de) & 1,1 & 4 & 72 & 0,4 & 2 & 28 & 1,4 & 6 & 100 \\
\hline Navarra (Com. Foral de) & 0,3 & 1 & 20 & 0,2 & 1 & 14 & 0,5 & 2 & 34 \\
\hline País Vasco & 0,7 & 4 & 48 & 1,0 & 4 & 68 & 1,7 & 8 & 116 \\
\hline La Rioja & 0,2 & 1 & 14 & 0,1 & 0 & 7 & 0,3 & 1 & 21 \\
\hline Ceuta y Melilla & 0,0 & 1 & 10 & 0,0 & 0 & 0 & 0,0 & 1 & 10 \\
\hline Total & $28,35 \%$ & 124 & 1.922 & $14,70 \%$ & 59 & 1.010 & $43,05 \%$ & 185 & 2.932 \\
\hline
\end{tabular}


ANEXO 2

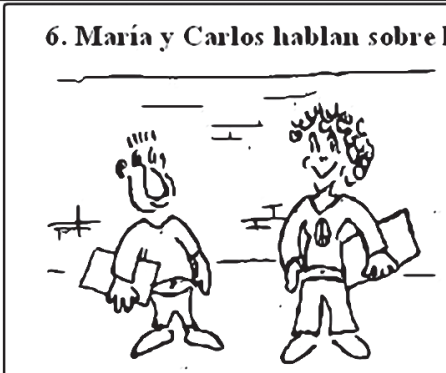

las Ciencias y la Tecnología

Maria: La mayoria de las cosas que hace la Ciencia son negativas para la Humanidad. Los científicos han creado armas -bombas, misiles, tanques...- para destmimos. Y otras veces han inventado productos que son mortales para las personas

Carlos: No estoy de acuerdo contigo. La Ciencia ha hecho que mejoremos nuestra salud. ¿Es que los medicamentos o las vacunas no nos permiten estar más sanos y vivir más tiempo? A demás, ¿quiénes crees que

han inventado aparatos -como los microscopios, rayos $\mathrm{X}$, termóm etros- que nos ayudan a curamos?

Di con quién estris más de acrierdo

Con los dos por igual $\square$

Más con Carlos que con Maria $\square$

Sólo estoy de acuerdo con Carlos $\square$
No estoy de acuerdo con ninguno $\square$

Más con María que con Calos $\square$

Sólo estoy de acuerdo con María $\square$

\footnotetext{
7. Un profesor pregunta a sus alumnos en clase: ¿Pensáis, de verdad, que las Ciencias son buenas para mejorar nuestra vida?.

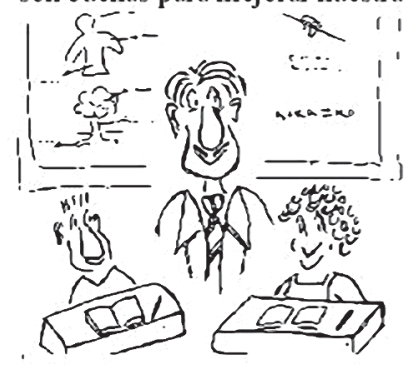

Carlos: Yo pienso que si. Gracias a las Ciencias, hacemos muchas cosas que nos gustan: oir música en la radio, ver películas en la tele, chatear con el ordenador, jugar con una consola.. Podemos viajar a otros lugares porque hay unos medios de transporte que, sin las Ciencias, no existirian...

Maria: Te veo muy optimista, Carlos. Yo creo que, con tanta Ciencia, vivimos una vida muy estresada. Cada vez somos más cómodos y más individualistas... Nos quedamos en nuestro cuarto con la tele y el vídeo, y no jugamos con los demás. Y de todo eso tiene la culpa la Ciencia.
}

\section{Di con cuíl estis más de acuerdo}

Con los dos por igual $\square$

Más con Carlos que con María $\square$

Sólo estoy de acuerdo con Carlos $\square$
No estoy de acuerdo con ninguno $\square$

Más con María que con Calos $\square$

Sólo estoy de acuerdo con Maria $\square$ 
8. Muchas veces encontramos noticias en los periódicos que hablan de consecuencias positivas de los descubrinientos científicos:

"Se descubre un gasóleo que rinde más y contamina menos", "Los nuevos molinos de viento: se abre un parque eólico que permutirá dar electricidad limpia a todos los pueblos del entomo", "Una mujer consigue tener una hija tras una fecundación in vitro"...

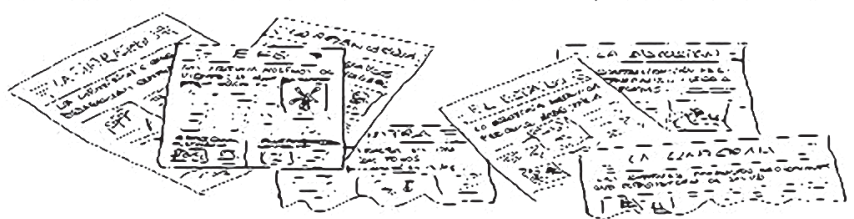

Pero también encontramos otras que no son precisamente positivas. Asi tenemos: "Las industrias petroquímicas producen una nube tóxica", "Los residuos que las fábricas vierten al Mediterráneo llegan a sus playas", "El agujero de la capa de ozono se triplica en menos de un año"...

En general, creo que en la prensu, en la radio o en la televisión:

Hay más noticias positivas que negativas sobre las Ciencias $\square$

Hay más noticias negativas que positivas sobre las Ciencias $\square$

Hay casi el mismo número de noticias positivas que negativas sobre las Ciencias $\square$

No sé si hay más o menos noticias positivas o negativas sobre las Ciencias $\square$

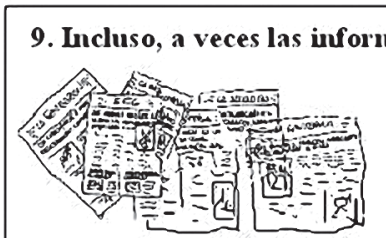

próximas"

Di con qué afinmación estás de acruerdo

Sólo me creo la noticia del primer periódico; es decir la positiva $\square$

Sólo me creo la noticia del segundo periódico; es decir la negativa $\square$

No me creo ninguna de las dos $\square$

Las dos noticias pueden ser ciertas $\square$

No sé qué periódico puede tener razón $\square$ 


\section{El otro día haciendo zapping por la tel e habia una tertulia de políticos}

Político 1: La Ciencia es sinónimo de progreso y eso es siempre positivo. Gracias al trabajo de los cientificos hemos avanzado a un ritmo impresionante. ¿Imaginaban nuestros abuelos la existencia de intemet, de las video-consol as o del AVE? Además, si reflexionáis un poco -cosa complicada en vuestro partido- os daréis cuenta que, debido a las Ciencias, han aparecido nuevas profesiones y también más oportumidades

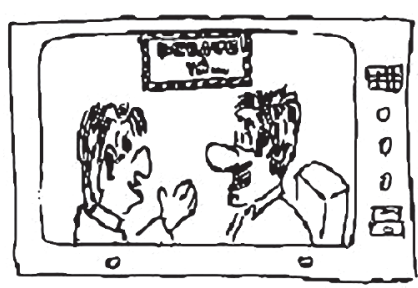

Político 2: Oye, no engañes a los que están viendo este programa. También las Ciencias han provocado grandes diferencias sociales entre los que pueden pagar la investigación cientifica y los que no. Eso ha provocado que los ricos se hagan más ricos y los pobres más pobres. Además, las Ciencias son cómplices del paro porque, por su culpa, se ha sustituido mucha mano de obra por máquinas.

\section{Dí con cuiál estís mis de acuerdo}

Con los dos por igual $\square$

Más con el Político 1 que con el 2 口

Sólo estoy de acuerdo con el Políticol $\square$
No estoy de acuerdo con ninguno $\square$

Más con el Político 2 que con el 1 व

Sólo estoy de acuerdo con el Político 2 口 


\title{
Primary and Secondary students' attitude towards the dichotomic view of Science
}

\author{
A. de Pro Bueno,A. Pérez Manzano \\ Universidad de Murcia
}

This research is part of a larger public opinion survey about Spanish children and teenagers' attitude. In it, we set out:

PP1. What stance do Spanish children and teenagers take when facing a number of dichotomies related to scientific products and their social effects?

PP2. Does their stance depend on their level of education, gender or type of school they attend?

We analysed some of the input received on attitudes and detected many meanings (concerning science and its discoveries, concerning scientists and their work, during a scientific activity, concerning scientific subjects, etc.) and that there seems to be some dependency on school variables (the level of education for instance) and on social variables (gender, the context, the Spanish autonomous community, etc.).

We then analysed the presence of science in the school curricula followed by the students of our study. We found that, during compulsory education, these attitudes have been addressed as teaching material (we are obviously unaware of how or how much they have been addressed), and therefore, the view students have of science and its discoveries should not be an "exclusive result" of the given context outside school (family, friend conversations, advertising, news, etc.).

We selected a cluster sample from official data, according to the Autonomous Communities, the level of education and the type of school. We used 6,827 questionnaires. The sampling error by level of education is $0.8 \%$ for primary education and $0.9 \%$ for compulsory secondary education. For the whole sample, the maximum sampling error is $0.01 \%$.

A PANA (Project on the Attitude of Children and Teenagers towards Science) questionnaire has been designed and applied for this study. In this work we have only provided the information on six of the questions asked: five dichotomies and a global evaluation. The first questions are related to "Health" (contributions to medicine vs. military industry), "Lifestyle" (life-style improvement vs. undesirable attitudes), "News" (positive news vs. catastrophes, disasters, etc.), "Credibility" (contradicting versions of the same news) and "Social repercussions" (progress, new professions, etc. vs. social problems). All of them were multiple-choice questions.

The results obtained concerning PP1 highlight that in the case of dichotomies, equidistant stances arise. This equidistance was not detected in previous works since the positive and negative effects were not compared in those cases. In fact, in the ROSE project (in the D questionnaires - environmental changes - and particularly in the G ones - my views on science and technology -, which are the most relevant questionnaires to this research), the students must give an opinion on an overwhelmingly positive set of statements. Would the same results be obtained in dichotomic situations? We do not think so. In fact, during our research, we have detected some differences between dichotomic results and those obtained from the "Global evaluation" (which is a lot more similar to the pro-scientific claims made in the abovementioned project). Hence, wouldn't it be better to supplement the information with dichotomic questions in order to know the students' real opinion?

In our case, the equidistant stances may be due to the intrinsic difficulty of what is involved, but above all, it has been detected that these debates have not been addressed in class during compulsory education. Nevertheless, there is some sort of a predisposition towards the positive (more frequent than the negative) - this trend may be to a lesser degree than the one found by Vázquez and Manasero (2009) - but in any case advantage should be taken of it.

On the other hand, regarding PP2, we have observed that the stances are more positive for students in primary education than for those studying their compulsory secondary education. Although this result is in line with other studies - as we stated in the first section - it is still worrying. In our case we have identified the student's stance after completing primary education, which is an important model since we do not know whether this has been identified in other studies with such a large sample as ours. Indeed, we found the largest differences in questions related to the media. This fact opens a new field of research - which is curiously quite different from what is usually studied during science class - and which has not been considered in previous studies.

Concerning gender, a certain tendency towards "a slightly" more positive view was observed in boys; however the differences detected clearly differ from some of the results of those studies mentioned in the first section. The differences weren't clear either in the study by Vázquez and Manasero (2009), when students had to adopt a clear position against some of the statements suggested. Furthermore, our study includes a field - "Health"- where girls show a more positive view about science. We believe that the gender dependency variable requires further research.

Concerning the type of school, we haven't found important differences supporting either type. In view of the results, within our educational context, there are probably some factors which have a more relevant impact on the stance taken by the students. Some have been detected and the rest, we will continue to search for. 